\title{
FEELING THE PAIN: DISRUPTIVE INNOVATION IN HEALTHCARE MARKETS
}

\author{
David J Williams \\ Loughborough University,UK, d.j.williams@lboro.ac.uk \\ Oliver Wells \\ Association of British Healthcare Industries,UK, oliwells@aol.com \\ Paul Hourd \\ LoughboroughUniversity,UK, p.hourd@lboro.ac.uk \\ Amit Chandra \\ Loughborough University, UK, a.chandra@lboro.ac.uk
}

\begin{abstract}
Healthcare is a growing market for products and services, costs are rising especially in the developed world. Disruptive innovations enable transition; less-skilled people do more sophisticated things in lower cost settings. In healthcare they promise to allow non-consumers new treatments reducing healthcare inequalities and ultimately to reduce the cost of individual treatments. A UK study shows adoption of such innovations is difficult because of the complexity of actors within the healthcare innovation network including clinical professionals, the supply chain, re-imbursement and regulatory agencies and healthcare service providers. Understanding cost containment in the overall system is key as is understanding the mechanisms and adoption conditions required to incentivise necessarily conservative service providers and clinical professionals with a busy, care driven agenda.
\end{abstract}

\section{INTRODUCTION}

There are many barriers to innovation in state-led healthcare systems including that of the UK: the busy care driven agenda of organizations and the individual; cost containment procurement strategies; the valley of death "chasm" between the early adopter and the early majority; the effect of regulation, clinical trials etc, etc,. This paper presents the perspective of a stakeholder group on the question "Can removing the barriers to the adoption of innovative technology - particularly disruptive technology - drive significant change in the NHS, the UK National Health Service, to the benefit of patients and to promote economic growth?". Consideration of this question should help to develop a better model for the creation of new products and consequently the introduction of a new technology into the NHS and other state healthcare systems and foster debate with healthcare commissioners about novel ways to overcoming the barriers to technology adoption including mechanisms for risk - clinical and commercial - and reward "upside" - sharing with the supply side. Understanding innovation in a complex network is at the core of this discussion. This network is made up of users, healthcare professionals, industry and other suppliers, regulatory and reimbursement agencies including healthcare commissioners - each of which feels pain of a different form: physical, professional or financial, that drives them to innovate. 
This keynote paper begins with an introduction to healthcare as a market in the UK context and innovation within healthcare with emphasis on the concept of disruptive innovation. The paper continues by reporting the results of an activity with a UK stakeholder group to determine a small number of interventions, based on a systemic view of the NHS and the other actors in the national innovation system, to encourage disruptive technologies that give opportunities for step changes in healthcare delivery performance and industry and economic growth.

\section{BACKGROUND}

\subsection{Healthcare as a Market}

Industry and business supplies healthcare delivery with the infrastructure, products and systems it requires. Healthcare is a growing market. In the western world it is important to recognize that the cost of healthcare delivery is rising, this is partially driven by changing patterns of demand, demographics, by the escalating costs of pharmaceuticals and the increasing costs of the opportunities of diagnostic instruments. These escalating costs demonstrate the scale and growth rate of the market, but bring with them the challenge of cost containment - rationing - and reducing operational costs. Regional inequalities in access to healthcare are also divisive - the western world has easy access to healthcare, this access is much more difficult in other less rich or privileged locations - this is also a challenge to business.

Healthcare is different from many other markets. The differences arise from the characteristics of the industry and its products and the range of professionals that work within it and the healthcare delivery system it serves. Two considerations shape the industry - products must be absolutely safe and ethical in their use - and much of the money that pays for them comes not from the patient that uses them, but from the healthcare system that pays the costs (the reimbursement system). The industry is carefully controlled by regulation and has to consider radical change thoroughly. People engaged in the industry come from the business and engineering professions, the life sciences including pharmacy, clinical practice and the caring professions. There are wide cultural differences between them, their backgrounds and their drivers. Business focuses on the market and finance reconciling complex conflicting objectives, engineers and physical scientists are driven to innovate in technology and have a quantitative approach, life scientists address complex biological science problems primarily from a qualitative viewpoint; clinicians and carers will always put patients - people - first.

\subsection{The UK Context, the NHS and Innovation the NHS}

The most significant healthcare provider in the UK is the National Health Service (NHS). The NHS was created in 1948 by the post war Labour government as the state system for the provision of healthcare with uniform, free access for all - a "universal service, based on clinical need, free at the point of delivery". Since then the NHS has grown to be one of the largest and most complex organizations in the world. There is also a complementary commercially oriented independent sector.

To the outsider and given the growing economic importance of healthcare this large and notionally integrated market place appears to be an ideal innovation opportunity for the UK economy and business to work with healthcare delivery to identify and create the new healthcare products both capable of success in world markets and of giving real 
patient benefit. This opportunity was formally recognized in the UK with the publication of the Cooksey Review (Cooksey, 2006).

Unfortunately innovation within this apparently favorable environment is not straight forward because of: the busy, care driven agenda of the organization and individuals; pressures on cost containment that drive procurement strategies to lowest cost, bulk buying and commoditization; continual policy and organizational churn as a consequence of political imperatives; capturing and communicating evidence and benefits across an organization of the scale and organizational complexity of the NHS; leadership and project management issues within a non-commercial care driven culture; the requirement to satisfy a regulator and for clinical trials or their equivalent; the larger than is usual "chasm" between the early adopter and the early majority as a consequence of the conservatism of clinicians and care delivery providers; as a consequence of industry structure, the significance of SMEs as technology providers to the innovation system; and the cultural gulf between industry and the NHS.

\subsection{Disruptive Innovation}

"Disruptive innovations enable a larger population of less-skilled people or providers with less training to do things in a more convenient, lower cost setting, which historically could only be done by specialists in less convenient settings" - Clay Christensen. (see Bower and Christensen 1995 and more particularly Christensen, Bohmer and Kenagy 2000).

Disruptive innovations are those that overturn apparently entrenched incumbent technologies. Innovations in the incumbent technologies have a product performance with time trajectory that begins to exceed the trajectory of the demand requirements of the customer. The incumbents then begin to be replaced by simpler cheaper, disruptive, innovations when the improvement trajectory of the disruptor allows its performance to match the lowest requirements of the demand. The most well known example of a disruptive technology in healthcare is less invasive medicine, particularly cardiac angioplasty and ultimately use of the stent (Hourd and Williams, 2007). Within the UK, Independent Sector Treatment Centres, which are commercially run, focused treatment centres operating in parallel to the mainstream NHS that take NHS patients, are another more service oriented instance of disruptive innovation. Disruptive technologies have three distinctive characteristics: they target non-consumers; they have a novel business model or value proposition and they drive out the incumbent in a niche where they deliver equivalent quality at a lower price.

Given the pressures on healthcare globally such approaches that have the potential to deliver radical benefits at lower cost and reduce inequalities are very attractive. As we will see, however, because disruptive innovations primarily target non-consumers, they will frequently increase overall system costs, while this is acceptable in purely commercial circumstances, it is harder to accommodate in an essentially finite resource state system there are no paying customers to drive change in state run health organizations.

\section{RESEARCH METHOD}

As indicated above disruptive technologies are anticipated to give opportunities for step changes in healthcare delivery performance and industry and economic growth. The object of the work was to deliver a stakeholder endorsed multi-perspective "Think Piece" - essentially this document - in order to promote and inform debate and identify areas that demand more attention. The stakeholders comprised a steering group that met three times 
in a workshop environment and a reading group to whom documents were circulated (group members are listed in the acknowledgements). The work is based on a systemic view of the NHS and the other actors in the national innovation system and attempts to build upon a virtuous circle linking improved healthcare delivery to requirements driven technology innovation. The work was process focused and did not attempt technology foresight or road mapping or address issues associated with professional practice. The work was presented for final review at the NHS Institute Conference at the Wellcome Trust on 5th November 2007.

\section{SUMMARY OF KEY RESULTS}

The results of the activity fall into four key areas: cost, price and value, system cost and value; the innovation process including risk and reward sharing; requirements capture and ideas generation; and forces on disruptive innovations.

\subsection{Cost, Price and Value, System Cost and Value}

A simple "Boston Box" for cost and quality as used in health economics when considering product price, Figure 1, is helpful in considering some of the micro-economic aspects of the innovation process.

The "box" helpfully defines areas where the case for technology has to be made (higher quality with higher cost); a "magic kingdom" of "no-brainer" decisions (higher quality and lower cost) and the "danger zone" of lower quality and lower cost. Making the case can be easier for new and radical technologies in the independent sector. This immediately identifies some of the challenges that the state system faces.

Importantly there is a perception that the national current commercial approach is pushing the NHS into the "danger zone" and that the NHS/healthcare delivery not benefiting as quickly as it should from products in the "magic kingdom". Underlying this is the recognition that disruptive technologies that have particularly targeted nonconsumers lead to increasing demand that can increase total system cost - and improve welfare - even if the technology is cost effective and of equivalent quality. This can be moderated by commercial demands for market share and consequent economies of scale leading to lower costs, and more careful consideration of system value through the life cycle. Total system cost increase may be moderated if resource is released by the substitution of the incumbent.

Technology adoption, diffusion and substitution is an intensely dynamic process. Importantly for the healthcare provider substitution and adoption requires the provision of two concurrent services and this is likely to favour the incumbent. Demand for the new technology or service may also be throttled by requirements for trials or post market surveillance. Consequently disruptive services must be introduced in partnership and show cost down and efficiency gains within a good understanding of capacity requirements. Training is key to effectiveness and reducing costs.

It is also important to recognise that legacy services within the system that have been impacted by the introduction of disruptive approaches will have to redefine their value proposition (for instance they may provide notionally the same service as the new alternative but actually deliver it to patients of multiple pathologies) to command increases in reimbursement tariff. System value must be the goal. As Porter and Tiesburg, 2006, has emphasised competing on results and value is the only alternative to shifting cost and limiting services - a zero-sum competition where one actor's gain is a loss for another. Silo thinking is not helpful! 


\subsection{The Innovation Process}

A simple model of the innovation process (Figure 2) is also helpful in bringing a number of issues to the surface. This model of the process recognizes the iterative nature of much healthcare technology development and that there are two dominant perspectives; that of the healthcare delivery system and that of business. It also highlights: the use phase; the identification of requirements from the use phase; the resourcing of the innovation process by multiple stakeholders - the risk and reward sharing process; the technology realization phase; and the healthcare assessment phase followed by adoption. In such systems ideas generation and requirements capture is complex and technology creation often comes from integrating several underpinning technologies which are emerging in parallel with different drivers. Comparative evaluation is complex, it must take place with the correct data and stakeholders including clinical benefit, regulation, reimbursement and purchasing; and take account of adoption conditions and definitions of quality, effectiveness and cost. Navigation of the regulatory pathways requires business to have access to a library of regulatory issues and signposting to experts. Note that it is considered that international regulation always favours the incumbent technologies.

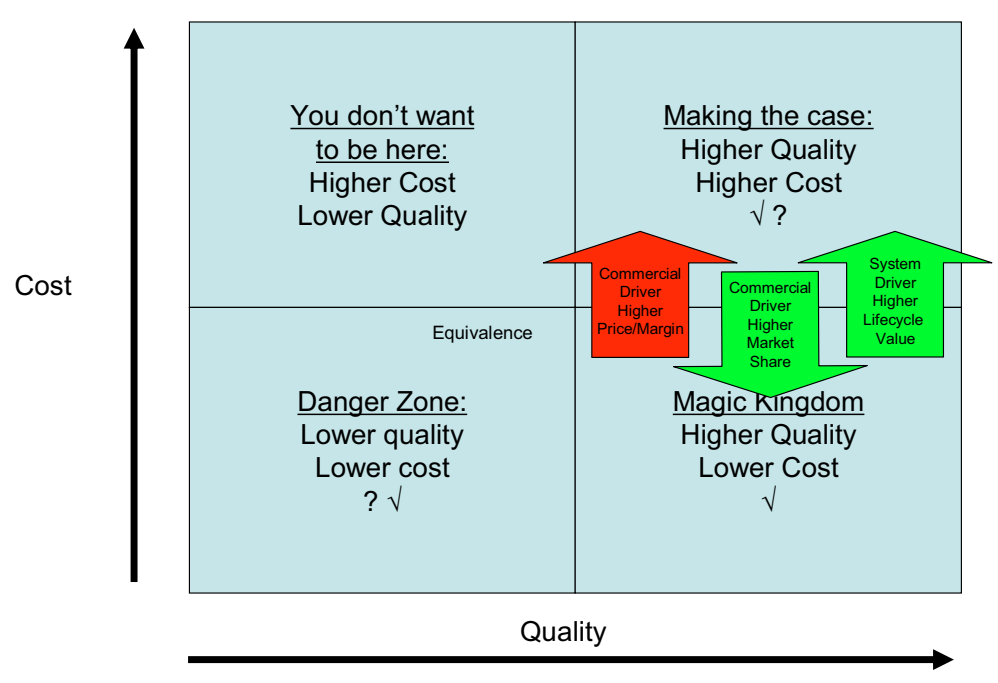

Figure 1. System Cost and System Value

It should also be noted that technology forms only a part of the innovation and that adoption requires matching all the acceptance criteria of the key stakeholders. Disruptive technologies involve much wider interactions than incremental changes. Users and beneficiaries will not be familiar with the technology. New skills may be required and supporting people may need retraining. There will be a lack of background knowledge of risks and benefits and the best ways to use the technology. Commercial organisations will also have to re-orientate their staff to sell and support the new technology

Feedback, attrition and building the evidence base takes place as the innovation cycles around the process. A major issue within the UK is the incentivisation of health professionals to participate in all of the process cycle. 
A key issue that must be recognized here is that, for business, the state national healthcare system, while large, is only part of the world market. Resourcing the innovation process and sharing financial risk and resource is complex with multiple actors including clinicians, industry; government; academics; venture and other commercial sources of funding and ultimately users (Hourd and Williams, 2006, 2008). It is clear that, even for UK based multinationals, the relative value of access to the NHS when compared with access to US clinical practice, is not sufficiently high. It is also clear that it is problematic for SMEs, especially potentially disruptive SMEs, to access the NHS (Hourd and Williams, 2008).

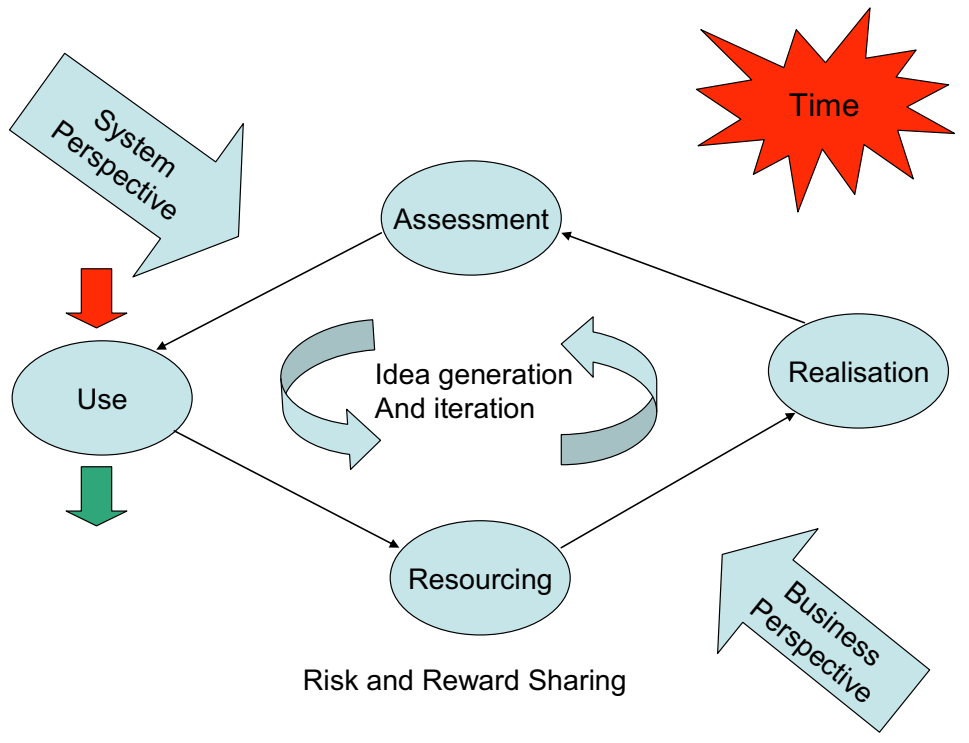

Figure 2. A simple model of the innovation process

To move forward on risk and reward sharing between industry and healthcare delivery we have to understand the commercial value of access/early access to the NHS and manage any risks of early access. Commissioning of health services should be a mechanism to incentivise innovation and may be able to address some of these issues. Disruptive technologies will require an improved understanding of the relationship between technology and service assessment processes and commissioning and reimbursement. This is just one instance of the "silo" based behaviour characteristic of large organisations that prevents actions that lead to better system value.

\subsection{Requirements Capture and Ideas Generation}

There are many sources for healthcare technology innovations. Some come from the inspiration of an inventor and their invention; some from an engineering tradition of problem solving. Some come from the user who has been personally touched by a healthcare problem or tragedy; some come from the healthcare professional and their demand for a more efficient tool to improve a clinical pathway; and some come from the healthcare provider and their requirement for reduced costs and service reconfiguration or 
a requirement to respond to the latest imperative from government. It all depends on who is feeling the pain and the kind of pain that they are feeling!

Figure 3 tries to capture some of the sources of innovations and the techniques, such as roadmapping, that might lead to a more systematic perspective on requirements capture balancing technology push with the identification of unmet needs. It is clear that the involvement of knowledge, practice and problem led healthcare professionals is required to ensure that innovations reflect a true projection of the current technology and requirements trajectory (Chatterji and Fabrizio, 2007). Given our earlier comments on incentivisation of health professionals, this involvement requires addressing in the current UK system.

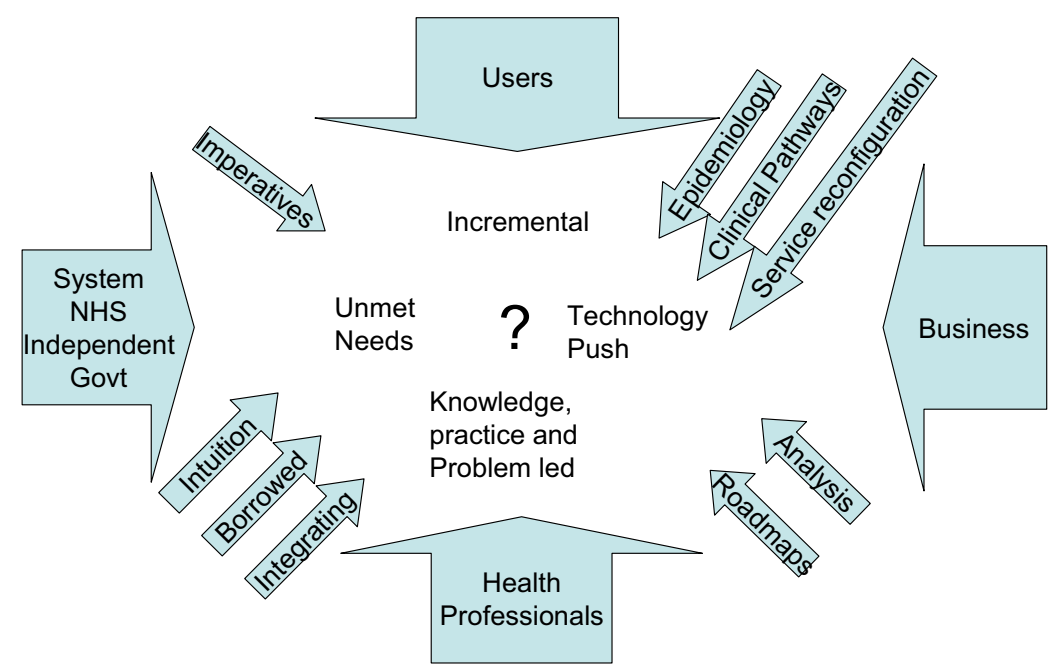

Figure 3. Requirements Capture and Ideas Generation

\subsection{Forces on Disruptive Innovations}

Figure 4 shows that the introduction of a disruptive technology requires the tensioning of four forces: the reaction force from the displaced technology and its suppliers; competitive pressures on the system and suppliers that drive innovation and invention; and user needs and benefits. It is intuitive that a focus on meeting user needs will stimulate improvement in performance and value. Clearly the tensioning of the four forces and the necessary responses will be associated with the level of disruption of the innovation, methods are therefore required to assess the disruptiveness of a technology in order to develop strategies to assist its adoption.

\section{CONCLUSIONS}

This paper has identified a number of key issues in a testing instance of innovation in networks. Disruptive technology adoption is dynamic, as are costs. Technology introduction alone is not sufficient, systems must change, these changes must happen in 
partnership to realize efficiency gains. For healthcare, targeting non- consumers delivers reduced inequality but inevitably drives up system cost even with lower product costs. This has two consequences: the requirement to consider the value of a technology through the whole system and lifecycle and the requirement for the incumbent technology or service to redefine its value proposition when challenged.

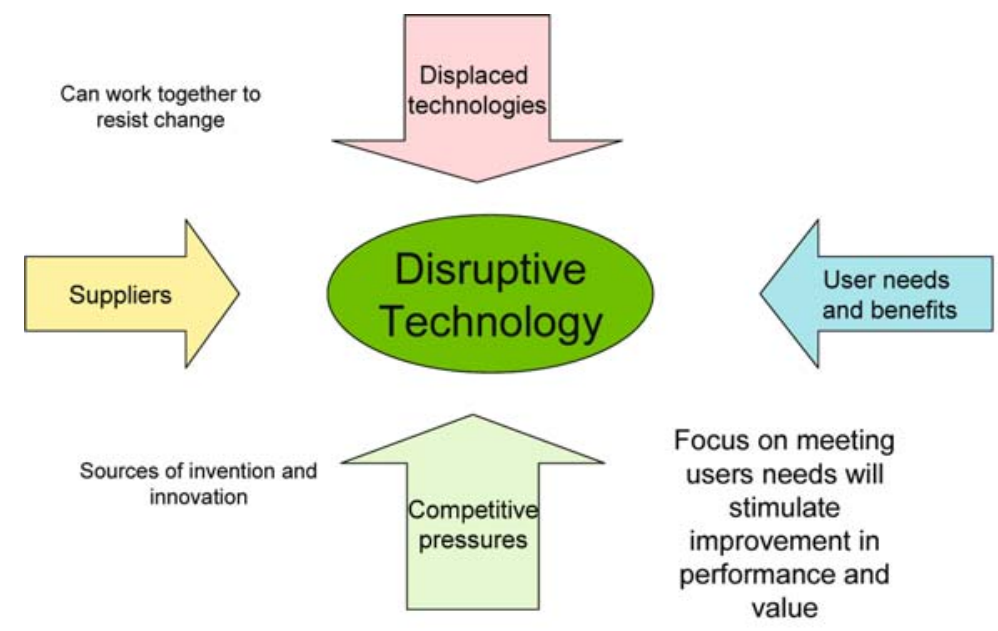

Figure 4. Disruptive Technology Forces

While there are a number of UK initiatives to promote heath technologies for patient benefit including the NHS Institute for Innovation and Improvement with its integral The National Innovation Centre, the Innovation Hubs (charged with exploiting innovations from within the health service) and the Adoption hub (charged with rolling out key promising innovations nationally), this analysis of the process of disruptive technology introduction in UK healthcare and particularly the NHS identifies some key areas for increased focus to prepare the ground for potential paradigm shifts:

- the creation of a process for the timely identification and quantification of realistic unmet needs;

- personal and organizational incentives (or perhaps reduced dis-incentives) to ensure continued involvement of clinical professionals in the whole of the innovation process;

- tools to profile the behaviors of key actors who are involved in adoption, and the development of matching strategies to overcome them;

- and, determining mechanisms for risk and reward sharing in the early introduction of technologies between the supply side and the NHS.

Access to the NHS for small and medium enterprises, SMEs, can be problematic and multinationals rarely see sufficient value from early access to the NHS and target other markets first. To move forward on risk and reward sharing we have to improve mutual understanding of the commercial value of access/early access to the NHS. This should form a core part of the evolution and realization of the healthcare service commissioning 
process to encourage and support the uptake of innovative technologies that are clinically effective and cost effective, a major topic of national debate, and current activities in the mapping of clinical value chains. This must include the impact of potentially increased system costs as a consequence of disruptive innovations that target non-consumers.

A recent review prepared by the HaCIRIC team at Imperial College (Barlow et al, 2007) on innovation diffusion and adoption processes in healthcare highlights that implementation is especially difficult when it changes the pattern of interdependence amongst individuals or groups and involves organizational and process change, this is inevitable with disruptive innovation. Complexity arises from the organization and its leadership; the organizational capacity and readiness for change; negotiation, power, micro-politics and local contexts; and evidence, risk and reward, perception and organizational norms. It also identifies orthodox micro-economic perspectives on innovation, such as ours here, ignore politics and power and the requirement to understand and incentivise dramatic, system level, innovation. This is another key area for focus in the future.

\subsection{Acknowledgments}

This work has been funded by the UK Engineering and Physical Sciences Research Council and forms part of the work of the Health and Care Infrastructure Innovation Research Centre, HaCIRIC, a collaboration of Imperial College and Loughborough, Reading and Salford Universities.

The work was carried out in collaboration with a stakeholder group, the Association of British Healthcare Industries (ABHI) and the UK NHS National Innovation Centre, part of the NHS Institute for Innovation and Improvement. Members of the stakeholder group included Richard Archer, Two BC; Chris Bantock and Bill Maton-Howarth, Department of Health; John Bessant, Imperial College; Clive Bray, MHRA; Kathryn Brown, BERR; Keith Chantler, Colin Morgan and Margaret Parton, Adoption Hub; Andrew Clark and Helen Hunt, EPSRC; Mike Craven and Steve Morgan, Nottingham University; Tony Davies, Medilink WM; Melanie Evans, Re:source Hub; Mark Fisher, Exomedica; Merlin Goldman, Technology Strategy Board; Michelle Hill-Perkins, Partnerships UK; Gareth Lloyd Jones, Medilink Yorkshire and Humberside; Geoff Morris, Medtronic; Clare Packer, Birmingham University; Tim Rubidge, Training Hub for Operative Technologies in Healthcare; Maire Smith and Brian Winn, NHS NIC; Mark Wickham, Smith and Nephew; John Wilkinson, ABHI; and Aniko Zaigon, Medicogen. We would like to thank this group for their active participation in discussions and review of documents. We would also like to thank Immanuel Sebastine for his help in the final reporting of this work. 


\section{REFERENCES}

1. Barlow J and The Imperial College HaCIRIC Team with Chiu K, Innovation diffusion and adoption processes in healthcare - a review of the Literature, Tanaka Business School Imperial College Internal Working Paper, December 2007.

2. Bower JL Christensen CN, Disruptive Technologies, Catching the Wave, Harvard Business Review 1995; Jan-Feb: 43-53.

3. Chatterji AK, Fabrizio K, Professional Users as a Source of Innovation: The Role of Physician Innovation in the Medical Device Industry, Working Paper Fuqua and Emory Business Schools, July 2007.

4. Christensen CN, Bohmer R, Kenagy J, Will. Disruptive Innovations Cure Healthcare? Harvard Business Review 2000; Sept-Oct: 102-111.

5. Cooksey D. A review of UK health research funding. HM Treasury Report. 2006, online at http://www.hmtreasury.gov.uk/independent_reviews/cooksey_review/cookseyreview_index.cfm.

6. Hourd PC, Williams DJ. Disruption in Healthcare, Angioplasty: An instance of disruptive innovation. Healthcare Engineering Working Paper RGC/74.1/07, 2007.

7. Hourd PC, Williams DJ. Success in Healthcare Technology Businesses: Coordination of the Value Milestones of New Product Introduction, Financial Stakeholders and Business Growth., Innovation: Management , Policy and Practice 2006; Volume 8(3): 229-247.

8. Hourd PC, Williams DJ, Results from an Exploratory Study to Identify the Factors that Contribute to Success for UK Medical Device Small \& Medium Sized Enterprises. Journal of Engineering in Medicine, Part H, 2008 (in press).

9. Porter ME, Teisberg EO, Redefining Healthcare: Creating Value-Based Competition on Results. Harvard Business School Press, 2006. 